\title{
PARADOJAS DEL MULTILINGÜISMO EN LOS U.S LATINO WRITERS
}

\author{
Ilse Logie ${ }^{1}$ \\ -Universiteit Gent, Gent, Belgica
}

\begin{abstract}
Resumen: Hoy en día, la movilidad y las relaciones interculturales resultantes de los procesos de globalización parecen fomentar el multilingüismo. También en el campo literario encontramos a menudo un discurso celebratorio al respecto, pero a pesar de que el multilingüismo goza de un mayor capital simbólico ahora, las prácticas reales de los autores latinos que viven en Estados Unidos sólo reflejan en parte tal hibridez lingüística y, de hecho, predomina una variante débil. En su estudio Beyond the Mother Tongue, Yasemin Yildiz explica por qué el paradigma monolingüe se convirtió en el habitus cultural de la modernidad y cómo consiguió oscurecer la existencia de otras formas de expresión lingüística. Basándose en ejemplos de la literatura alemana, Yildiz percibe, sin embargo, una evolución hacia una nueva condición, la condición postmonolingüe, que reconoce la validez de las prácticas multilingües. En este trabajo, que presenta una investigación en curso, me centraré en cuatro textos autobiográficos recientes escritos por autores que residen en Estados Unidos y que tienen el español como uno de sus idiomas, ya que se supone que es en la autobiografía donde más explícitamente engarzan las elecciones lingüísticas con la construcción de la identidad. Mi objetivo es arrojar luz sobre las paradójicas estrategias através de las cuales estos Latino Writers forjan una alternativa al binomio monolingüismo/multilingüismo.
\end{abstract}

Palabras clave: Multilingüismo; Autobiografía; Estudios Latinos; U.S Latino Writers 


\title{
THE PARADOXES AND PITFALLS OF MULTILINGUALISM IN U.S LATINO WRITERS
}

\begin{abstract}
Terms such as "globalization" and "World Literature" suggest that multilingualism rather than monolingualism is the sign of the present times. Celebratory discourse in Literary Studies seems to confirm this trend in Academia. In spite of the increased symbolic capital of the multilingual, real literary practices of authors with Latin American roots living outside Latin America only partially reflect this hybridity. "Soft multilingualism" is rather the norm. Analyzing examples of German literature, Yasemin Yildiz (Beyond the Mother Tongue) identifies this contradiction between multilingual realities in society and the persistence of the monolingual paradigm in literature as "the postmonolingual condition". In my article, in which I present an ongoing research project, I will focus on four contemporary intercultural autobiographies where language choices are explicitly connected to questions of identity. By doing so, I intend to shed light on the paradoxical strategies through which bilingual authors (English and Spanish) in the U.S. shape their struggle towards the postmonolingual paradigm.
\end{abstract}

Key Words: Multilingualism; Autobiography; Latino Studies; U.S. Latino Writers.

\section{Premisas de la investigación}

Cuando, con An Van Hecke (KULeuven), formulamos el proyecto de investigación "Vivir en la traducción: las paradojas de la escritura autobiográfica multilingüe latinoamericana y latina 1980-2015", ${ }^{1}$ nos proponíamos rastrear las manifestaciones del multilingüismo en escritores radicados en América y Europa que tenían el español como uno de sus idiomas principales. Para que el corpus resultara manejable, nos limitamos a combinaciones de idiomas europeos (inglés, francés, alemán, portugués) sin tener en

\footnotetext{
${ }^{1}$ Este artículo se escribió en el marco del Proyecto G046117N "Lives in translation. The Paradoxes of Latin-American and Latino Multilingual Autobiographical Writing 1980-2015" financiado por el FWO Flandes.
} 
cuenta lenguas indígenas, y nos impusimos una restricción genérica: sólo incluiríamos textos autobiográficos (en el sentido amplio del término). Optamos por autobiografías porque en este tipo particular de textos la elección del idioma se vincula, mediante operaciones literarias, con cuestiones de identidad. Planteamos preguntas del tipo: ¿Cómo abordan los textos autobiográficos la conexión entre las diferentes lenguas en juego (lengua materna, lengua adoptiva, a veces una tercera lengua) y la identidad, entendida esta última como construcción discursiva? ¿Cuál es la influencia del contexto y de la jerarquía percibida entre las lenguas? ¿La vida en un entorno de lenguas extranjeras ha provocado una pérdida de identidad o ha permitido la creación de un nuevo yo?

La premisa que sustentaba el proyecto era que el exilio, la migración, la emancipación postcolonial y la globalización habían dado lugar a un aumento del número de textos literarios multilingües en la literatura latinoamericana y latina desde 1980. Esta hipótesis venía respaldada por el "giro multilingüe" que se perfiló, desde la década de los 90, en la literatura comparada. Según, por ejemplo, Emily Apter o Rebecca Walkowitz o, para América Latina, Doris Sommer, la literatura escrita en más de un idioma, aunque lejos de ser un fenómeno nuevo, está en ascenso y ha dado un salto cualitativo en las últimas décadas.

Con el fin de reducir aún más el alcance del proyecto, y teniendo en cuenta la trayectoria de nuestro grupo de investigación, decidimos centrarnos en la literatura mexicana y mexicano-americana y la del Cono Sur respectivamente, y dejar fuera, al menos en una primera etapa, la obra de autores de origen caribeño. Lo que volvió tan interesante la comparación de las dos áreas seleccionadas es que los escritores de origen mexicano mantienen una relación sustancialmente diferente con el inglés. Al compartir México y Estados Unidos una frontera y una historia llena de fricciones, el inglés, para los autores mexicanos y mexicano-americanos, nunca puede ser un idioma neutro, sino que se ve atravesado por tensiones ideológicas. Los autores provenientes de Argentina y Chile, por su parte, tienen una larga tradición de "extraterritorialidad" (según el 
conocido término de Steiner) en la que Europa ha sido el principal punto de referencia. La actitud hacia el inglés de esos autores conosureños que emigraron a EEUU (Estados Unidos) es, por tanto, mucho más distante; la gran mayoría nunca ha abandonado el español como lengua de escritura.

La producción "latina" es de suma importancia para este proyecto. Esto también explica por qué decidí centrar mi artículo en algunos aspectos de este subcorpus ya de por sí heterogéneo. El multilingüismo presente en los escritores latinos seleccionados para este trabajo se debe a motivos diversos. Los autores argentinos y chilenos del corpus son, en su mayoría, representativos de dos olas migratorias: la primera, ya bastante trabajada por la crítica, llegó a EEUU en la década de los 80 por motivos políticos, huyendo de las juntas militares de Pinochet y Videla. Para Manuel Puig, Sergio Waisman o Ariel Dorfman, el español es tanto el idioma en el que se expresa la nostalgia como la lengua que encarna al régimen represor. La segunda ola se vincula más bien con los procesos de (re)democratización posteriores a 1990. Para estos autores (Lina Meruane sería un ejemplo representativo), la decisión de vivir en el extranjero y manejar varios idiomas se produce en un ámbito de libertad y está inscrita en un proyecto de autorrealización personal.

El caso de los autores mexicanos, y en particular de los mexicano-americanos, tiene que remontarse mucho más atrás en el tiempo, hasta el movimiento chicano de los años 60, e, históricamente, hasta la anexión que llevó a cabo EEUU de parte del territorio mexicano en 1848. Una diferencia fundamental con otras comunidades latinas es que muchos chicanos no han pasado por la experiencia migratoria. Los primeros autores chicanos (Tomás Rivera, Rudolfo Anaya, Rolando Hinojosa, Gloria Anzaldúa) escribieron sobre EEUU desde una perspectiva de resistencia contra la colonización interna. Estos autores se oponían al monopolio del inglés escribiendo en español (Rivera) o combinando inglés y español (Sandra Cisneros). Otros, al contrario - los menos - se rebelaron contra sus comunidades de origen escribiendo en inglés (Richard Rodriguez). En las últimas décadas, se ha ido produciendo 
la transición de una literatura mayormente en español a una en la que predomina el inglés, porque han recibido su educación formal en este idioma. Se observan grandes divergencias en el modo de interpretar esta evolución. Si para algunos ha comenzado un proceso de autoborrado y de comodificación acerca de cómo debe expresarse un autor chicano, otros subrayan que la pervivencia de la biculturalidad se plasma en recursos literarios más oblicuos. Sea como sea, es indudable que los autores chicanos experimentan, en creciente medida, una presión para asimilarse a un tipo singular de literatura, lo que significa que la etiqueta se ha convertido en una "marca" estereotipada por el mercado ante la cual conviene posicionarse (véase al respecto la evocación irreverente de la "trampa" chicana en How to be a Chicana Role Model de Michele Serros, que ya data del 2000).

Mi estancia en Nueva York ${ }^{2}$ me hizo comprender, asimismo, que sería imperdonable dejar de lado una tercera área geográfica: los autores originarios de las tres islas del Caribe de habla hispana; no sólo porque, numéricamente hablando, son el segundo grupo más importante en EEUU después de los mexicano-americanos, sino también por la movilidad fluida que caracteriza a sus comunidades y por su papel pionero en la creación de la literatura latina. En especial los autores puertorriqueños - que están en una posición única, ya que como residentes de EEUU se los considera estadounidenses e inmigrantes a la vez -, pero también los cubanos y dominicanos.

Me di cuenta, además, de la especificidad de la experiencia latina, aunque era casi imposible definirla como categoría. En las últimas décadas, la proyección de la comunidad latina se ha extendido a todos los aspectos de la cultura estadounidense. Esta visibilidad va acompañada del riesgo de una recuperación institucional, a la que se ha llamado "tropicalización hegemónica" (Sandoval y Aparicio

\footnotetext{
${ }^{2}$ De agosto a diciembre del 2018 tuve la oportunidad de disfrutar de una estancia en la Fordham University de Nueva York, lo que me permitió trabajar con estudiantes latinos y entrar allí en contacto con la escena cultural.
} 
675). Oficialmente, el rótulo "latino" se refiere a las personas de ascendencia latinoamericana nacidas o criadas en EEUU, o que viven allí. En la entrada "Latino, Latina, Latin@"3 de Keywords for American Cultural Studies, Juana María Rodríguez ofrece la siguiente definición de "Latino": "designación étnica que distingue a los latinoamericanos que viven en Estados Unidos de aquellos que viven en sus países de origen" (2). En su revisión de los debates académicos sobre el término, Laura Loustau (31-42) destaca el carácter construido y arbitrario de este significante borroso que, desde entonces y ante el clima antiinmigratorio exacerbado de la era Trump, se ha complejizado aún más, si bien al mismo tiempo la comunidad muestra mayor unión. Pese a que ya son 60.000 .000 y representan el segundo grupo étnico de EEUU, nadie - me enteré durante mi estancia en New York - está realmente seguro de a quién se califica exactamente como tal y a quién no, ni qué es exactamente la literatura latina; hay que declararse latino para serlo, en un gesto político performativo de toma de conciencia identitaria moldeado por el activismo.

Entre latinos, se debate con vehemencia qué tipo de memoria histórica permite ciertas reivindicaciones y cuál convierte en ilegítimas otras. En su contribución al Companion to US Latino Literatures, el escritor argentino-estadounidense Sergio Waisman se interroga sobre la legitimidad de la inclusión de escritores argentinos como él, que llevan años escribiendo desde EEUU aunque sea en español, en la categoría de los escritores latinos (158). Waisman, defiende tal inclusión y observa que los temas de identidad y lenguaje en la literatura latina de EEUU son arduos, mientras que la categoría del propio "latino" está en un proceso de constante transformación. El escepticismo de Waisman se ve reflejado en la organización de los departamentos académicos de EEUU, donde en los "Latino Studies" raramente se incorporan obras de escritores chilenos o argentinos, pese a que se podría

${ }^{3}$ En septiembre 2018, el diccionario Merriam-Webster registró “Latinx” como variante neutral en cuanto a género. 
ganar mucho, al decir de Waisman, si se entablara el diálogo entre estos dos campos concebidos como compartimentos estancos: US Latino Studies por una parte, y Estudios Latinoamericanos por otra.

De acuerdo con esa misma lógica institucional, comprendí que nuestro proyecto, que combina literatura mexicana y mexicanoamericana, provocaba resquemores entre quien trabaja literatura chicana porque era como hacer desaparecer la especificidad de su campo y subsumirla en la literatura mexicana, cuando desde México no se asume esa tradición como propia. En una de las conferencias que di, hablé de Los niños perdidos de Valeria Luiselli y de la compleja génesis bilingüe de ese texto. El debate que siguió giraba en torno a la pertenencia de Luiselli: escritora ¿mexicana o mexicano-americana, gringa?

Como señala Aparicio (368), es obvio que hoy en día cada vez más escritores latinos provienen de experiencias sociales y antecedentes educativos que difieren de las identidades marginales de los escritores de los 60 y 70. Entre los que ganaron voz a partir de los 90 están muchos inmigrantes recientes de America Latina, como aquellos cuyas historias se recogen en Se habla español: voces Latinas en USA (United States of America) (Edmundo Paz Soldán y Alberto Fuguet). Este tipo de antologías puede verse como un intento de llamar la atención sobre el creciente número de autores latinos que de nuevo optan por escribir en español, enraizados en experiencias intelectuales y cosmopolitas de clase media o clase media alta; autores bilingües que principalmente han estado afiliados a universidades estadounidenses y han llegado con una beca o un profesorado (Luiselli, pero también Lina Meruane, Yuri Herrera, Eduardo Halfon, Claudia Salazar...). Su llegada ha puesto en marcha un proceso de identidad transnacional muy diferente de las reivindicaciones de identidad de los latinos en el pasado, y señala la difusión global de la literatura latinoamericana y la globalización de la Latinidad.

Surgió entonces la siguiente pregunta: ¿se pueden eludir esas nebulosas identitarias y académicas estudiando posibles configuraciones del multilingüismo en algunos textos recientes 
(posteriores al 2000) del subcorpus? Si la crítica tiende a asociar lo latino con lo híbrido y si muchos textos latinos mezclan hasta cierto punto el español y el inglés, a primera vista el spanglish parece contener la clave de la expresión latina.

El spanglish es parte del dinámico contexto plurilingüe en el que viven los latinos en EEUU. El spanglish y otras manifestaciones híbridas son tan relevantes porque niegan la idea de que se pueda trazar una clara línea de demarcación entre las lenguas, una suposición que se desprende de términos tales como "primera lengua", "segunda lengua", "hablante nativo". Tidigs y Huss, que a su vez se basan en los aportes teóricos de, entre otros, Naoki Sakai y Till Dembeck, han demostrado que estas etiquetas, antes de describir la complejidad del uso de la lengua, cumplen una función ideológica (Tidigs \& Huss 217-219). Lipski, que define el spanglish entre otros como la intercalación fluida y frecuente del español y el inglés en una sola conversación u obra literaria (9), a veces dentro de la misma oración, explica que tal cambio de código responde principalmente a factores pragmático-discursivos y se debe sólo en menor medida a la falta de competencia lingüística en una de las lenguas en juego. De esta manera, el lenguaje híbrido no sólo tiene integridad, sino que despliega potencial expresivo y agrega profundidad a la voz del artista. El uso consciente del cambio de código es en sí mismo un acto político: refleja los conflictos creados por el contacto entre las dos culturas o recalca su plusvalía. Esto ya era el caso en los primeros discursos postcoloniales, piénsese en textos frecuentemente antologizados como Borderlands/La Frontera (1987) de Gloria Anzaldúa. Sin embargo, el propio concepto de "código" presupone la existencia de sistemas lingüísticos delimitados, por lo que resulta más interesante definir el cruce lingüístico como el encuentro verbal entre las culturas anglo e hispana, como práctica vital que varía de acuerdo a la región, ya que la comunidad latina tampoco puede presentarse como una entidad monolítica. No sólo se trata de lenguaje, sino también de forjar otros imaginarios y tradiciones literarias. 


\section{Posibles configuraciones del multilingüismo}

\section{Fusión linguiística cosmopolita: Susana Chávez-Silverman versus Ilan Stavans}

Los primeros resultados de los análisis iban en contra de nuestras expectativas. Las narrativas autobiográficas que leímos incorporan el español a algún nivel, pero no en cantidades significativas. Tomemos On Borrowed Words, las memorias de Ilan Stavans, el escritor mexicano que ahora es profesor en EEUU y un ferviente defensor del spanglish. Si bien la matriz de su libro es claramente multilingüe, estas otras lenguas - el español, el yidis y el hebreo aparecen sobre todo como latentes sin que el autor explote a fondo la potencialidad que encierra la creación idiomática. Se capta al yo diaspórico mediante otros recursos, como la tematización del metalingüismo y procedimientos intertextuales que se dirigen al lector monolingüe estadounidense. De este modo, On Borrowed Words contiene un desafío muy moderado a la cultura hegemónica norteamericana. Nos encontramos aquí ante una primera paradoja: a pesar de que el multilingüismo se ha convertido en el nec pus ultra del capital simbólico según el propio Stavans, lo que hacen muchos autores es añadir ciertos acentos extranjeros (elementos culturalmente reconocibles como citas literarias o realia) a textos estructuralmente monolingües. Así, en su lucha por el reconocimiento, cumplen con las expectativas sin dar la espalda a las normas literarias estadounidenses; la forma en que lo hacen ha sido tildada por David Gramling de "estratégicamente centrípeta" (135).

Un contra-ejemplo espectacular, en el que la mayoría de las frases son híbridas hasta el punto de carecer de una lengua básica, es Killer crónicas, Bilingual Memories de Susana ChávezSilverman. Chávez-Silverman recibió una educación multilingüe y multicultural y ha vivido en varias ciudades, entre las cuales figuran Los Ángeles, Madrid, Buenos Aires y Guadalajara. Sus memorias Killer crónicas, publicadas en el 2004, surgieron de la necesidad de viajar y de negarse a traducir una vida multicultural 
a un solo idioma y un solo acento. Mezclando el inglés, diferentes inflexiones del español, el portugués, el italiano y el afrikaans a nivel léxico y sintáctico, la autora opera una verdadera fusión y forja una poética que trasciende lo nacional, representando así la realidad de la globalización. El carácter profundamente multilingüe de su texto la sitúa en una larga tradición de resistencia latina al inglés en EEUU. Pero lo que distingue esta crónica de otros textos latinos es que, además, forja conexiones con otras naciones y otros continentes que no sean América. El español se convierte en una lengua sin fronteras, sin por ello dejar de estar localizada. Aunque la experimentación lingüística no es nueva en los autores latinos (ya en 1971, Tomás Rivera escribió Y no se lo tragó la tierra en el dialecto coloquial que era típico de los trabajadores migrantes mexicanos en el suroeste), Chávez-Silverman ha llevado la interpretación bilingüe a un nivel completamente nuevo, en esto solo comparable con Yo-Yo Boing de la puertorriqueña Giannina Braschi. Así es como Chávez-Silvermann se dirige a sus lectores en su prefacio:

These crónicas began as letters: cartas a amigos extrañados, love letters to cities, smells, people, voices and geographies I missed. O, por otra parte, comenzaron como cartas a un lugar or to a situation that I was experiencing intensely, casi con demasiada intensidad and yet pleasurably as well, a sabiendas de que la vivienda acabaría demasiado pronto. Cierro esta glossary crónica then - para voh, para ti, for you the reader - with an invitation to dive into este mi texto intersticial. Go on, lánzate. Lance yourself. (ChávezSilvermann, "Glossary Crónica” XXI)

\section{El multilingüismo: ¿un mito literario?}

Por radical que sea, la mera existencia de una obra como Killer Crónicas indica que está cobrando forma algo parecido a 
un "paradigma postmonolingüe latino". En Beyond the Mother Tongue, su monografía sobre la literatura alemana, Yasemin Yildiz comenta la crisis que sufre la exclusividad del vínculo entre lengua y experiencia; o sea, el cuestionamiento del paradigma monolingüe; una construcción histórica que data de siglo XVIII, pero que sigue estructurando la vida cultural hasta el día de hoy. Yildiz está convencida, sin embargo, de que la creciente movilidad plantea la posibilidad de un nuevo paradigma, al que ella llama "postmonolingüe", que reconoce la existencia de prácticas multilingües. Su concepto de "condición postmonolingüe" identifica precisamente la tensión entre la persistencia de la norma monolingüe, por un lado, y la intensificación de las prácticas multilingües, por el otro. Las prácticas y actitudes postmonolingües son aquellas que desnaturalizan la conexión entre la lengua "materna" y la identidad de un sujeto hibridando la primera, la segunda o ambas.

¿Por qué, entonces, Killer Crónicas nos aparece como un ejemplo extremo en el panorama estadounidense? En In Babel's Shadow, Brian Lennon sostiene que el espíritu multilingüe contemporáneo no sólo choca con la persistencia del paradigma monolingüe, sino también con las leyes implacables de la industria editorial, especialmente en EEUU. Basándose en un artículo de Yaseen Noraani, distingue entre textos multilingües "débiles" y "fuertes". Describe la primera variante como una reproducción de la relación entre la lengua dominante y una lengua minoritaria (Lennon 83). El multilingüismo "débil" mantiene el idioma extranjero en palabras y frases individuales, lo etiqueta o traduce, como ocurre en el caso de On Borrowed Words. La lengua extranjera viene en una dosis tan pequeña que sólo ofrece "un toque de verosimilitud cultural" que sazona el texto sin dañar su forma doméstica (Lennon 10, mi traducción). Pero incluso esta opacidad mínima se considera como innovación o hibridación, aunque principalmente reconfirma el estándar. Según Lennon, lo que los lenguajes racionalizados de la publicación de libros no pueden tolerar es una desviación radical de esa norma. No nos debe extrañar, por tanto, que la obra de Chávez-Silverman se haya publicado en una editorial académica 
y se haya estudiado casi exclusivamente en contextos académicos. Gramling formula un diagnóstico similar cuando advierte que no hay que sobreestimar la disponibilidad de los lectores para leer textos multilingües y, aún menos, la voluntad de los editores para adaptarse a la diversidad lingüística (Gramling 129). La tesis de Gramling es que, aunque las lenguas están más a menudo en contacto entre sí, de hecho coexisten principalmente, sin interacción profunda.

\section{Juan Dicent: la voz interlingual}

Pero cuando pensamos en el típico escritor latino que practica la alternancia de códigos, los nombres que surgen espontáneamente son diferentes del de Stavans o Chávez-Silverman. El caso más célebre de la creación de una "interlengua creativa" (Bruce-Novoa 10) a través del spanglish es sin duda el del dominicano-americano Junot Díaz, cuya lengua de base es el inglés. Tanto en Drown (1997), su primera colección de relatos, como en su novela The Brief Wondrous Life of Oscar Wao (2007), ambos publicados por la editorial norteamericana mainstream Riverhead Books, el uso desestabilizador del español sirve como vehículo para la expresión de un mundo fragmentado. En un esclarecedor artículo, Rita De Maeseneer define la praxis de Díaz como pionera porque se opuso a la domesticación de voces de otra procedencia. De Maeseneer destaca sobre todo el principio de la no-traducción (la "assertive nontranslation" de Ch'ien (209)) que consiste en la inserción de frases y sintagmas en español, la práctica de diversos tipos de cambio de código y la tolerancia hacia "errores" debidos a un aprendizaje oral del español dominicano. (De Maeseneer, 350-351).

Se trata, sin embargo, de un multilingüismo estilizado y controlado, preocupado por la legibilidad. En esa misma vertiente se inscribe la obra de Juan Dicent, figura más underground. Nacido, como Díaz, en la República Dominicana, Dicent reside en New York desde 2007, por lo que su espacio de enunciación "posinsular" (Néstor Rodríguez citado en Bustamante 122) le permite desarticular tanto el topos de una República Dominicana de 
sol y playas como el imaginario del país de acogida, EEUU. Poeta, cuentista y blogger, tematiza, en obras que salen publicadas en el circuito de las pequeñas editoriales independientes, la emigración a EEUU por medio del uso sostenido de la oralidad, la ironía y un potente bilingüismo con el español como lengua básica. Los breves e irreverentes relatos recogidos en los tres tomos de su saga testimonial que ya han salido hasta ahora - Summertime (2006), Winterness (2012) y AutumnFalltime (2018) - cuentan anécdotas de la cotidianidad precaria de los dominicanos en EEUU: su espanto ante la primera nieve, cómo bregan con el frío, las problemáticas relaciones con la familia dejada atrás, la difícil inserción en el mercado laboral. Logran reproducir, con gran economía de medios, el intercambio constante de personas, mercancías y cultura inmaterial entre ambos lugares, al tiempo que describen el surgimiento de un nuevo yo, que acaricia una versión bastante sui géneris del sueño americano y no se deja ceñir a imaginarios homogéneos de identidad.

\begin{abstract}
Southern Comfort
Y si la gente puede divorciarse de un cónyuge al que se ha dejado de amar, ¿por qué no puedo yo divorciarme de mi familia? Salir por esa puerta, tomar un avión en La Guardia hacia Memphis, visitar Graceland; subirme en una guagua hacia Chattanooga. Allí buscarme una white trash con cara de batata, gorda, que me dé golpes todas las noches; vivir con ella en un trailer izando orgulloso la bandera confederada mientras toco en un banjo Star-Spangled Banner; descubrir la felicidad simple de cultivar un jardín de mufflers, mullets y escopetas. Ser mordido por una serpiente mocasín. (Dicent, Winterness 39)
\end{abstract}

Dicent da a sus textos títulos en inglés e incluye giros y frases en ese idioma, cuestionando de esta manera la lengua vehicular de la comunidad dominicana, que ya deja de ser de un modo tan claro el español. Pero lo más impactante es que su escritura se sirve 
de al menos dos sistemas de referencias culturales: su tierra natal caribeña y la escena latina en EEUU. Si su proyecto contribuye a la realización del paradigma postmonolingüe, se debe sobre todo a que sabe congregar de un modo particular ambas tradiciones culturales sin caer en las metáforas conceptuales de "fronteras", "puentes" y "guiones" que prevalecen en las lecturas críticas de la producción artística latina. No solamente en la superficie, es decir, en cierto pintoresquismo exótico practicado por muchos de sus predecesores (el “español transparente" evocado por Lourdes Torres 79), sino por estar ubicado en la intersección de estas culturas y fundir ambas maneras de situarse en el mundo, sin que esto implique ausencia de tensiones ni eclecticismo gratuito.

\section{Sergio Waisman: alternancia y autotraducción}

Volvamos ahora a los interrogantes de Sergio Waisman: ¿Hay que dejar fuera a los escritores de origen argentino que viven y trabajan en EEUU por la posición más periférica que ocupan dentro de la constelación latina? ¿O el descentramiento lingüístico puede manifestarse de otro modo, sin exhibir necesariamente las marcas más llamativas que se suelen asociar con el multilingüismo, como el cambio de código? Rainier Grutman define el multilingüismo como "la copresencia de dos o más lenguas en una sociedad, un texto o un individuo determinado" (182); o sea, que puede tipificar una trayectoria autorial más allá de un texto específico. En el caso de Waisman, por ejemplo, cabe hablar de monolingüismo serial porque el autor publica sucesivamente en sus dos idiomas. Pero si, como sostiene Grosjean, un sujeto multilingüe no es la suma de dos monolingües, sino que se caracteriza por "una configuración lingüística única y específica" (76), tal configuración no puede dejar de afectar a los textos que produce. La activación constante de dos o más idiomas influye en el proceso de escritura, una visión que nos hace entender las similitudes entre la literatura multilingüe escrita a través del filtro de un idioma extranjero y la escritura multilingüe en el idioma materno del autor, si es que esos conceptos aún tienen vigencia. 
Según esta lógica, convendría abogar por replantear el multilingüismo, que vendría a designar no sólo la presencia literaria de dos lenguas, sino también los ecos de otra lengua y cultura detectados en la llamada prosa monolingüe. Conforme este replanteamiento, escritores que residen en EEUU pero cuya lengua de escritura es el español sin code switching, como Sylvia Molloy, Edmundo Paz Soldán o Sergio Chejfec, son perfectamente capaces de producir textos multilingües. Un enfoque más productivo de la literatura multilingüe sería, por tanto, considerarla no como una división (mono/multi) sino como un espectro gradual; en otras palabras: subrayar la variación lingüística potencial de todo texto, en lugar de presentar la literatura multilingüe como una categoría aparte, y como una desviación de una norma monolingüe bien establecida. O sea, naturalizarla.

Detengámonos ahora en la autoficción Leaving (2004) del traductor Sergio Waisman, una exploración de su identidad profundamente plural. Waisman tenía ocho años cuando sus padres emigraron de Argentina para instalarse en EEUU. Leaving es una colección de meditaciones retrospectivas altamente reflexiva, que raya en lo experimental. La trama arranca en una cocina de Colorado, Estados Unidos, donde una brisa le hace creer por un instante al narrador que está en Buenos Aires, a la orilla del Plata, donde es verano. Pero, cuando mira por la ventana, se da cuenta de su error, puesto que hay nieve todavía en las montañas. Desde este presente impregnado de lejanía se inicia un viaje íntimo donde el protagonista trata de recuperar escenas clave de su familia, una familia judía que tuvo que exiliarse de Europa del Este debido a la Segunda Guerra Mundial y que, finalmente, se desperdigó por Nueva York, Buenos Aires y Florida. Debido a que lleva tanto tiempo viviendo en EEUU, el narrador siente que tiene un mejor dominio del inglés que del español, aunque este último permanece inextricablemente ligado al mundo afectivo de su infancia. Es, sin embargo, otro tipo de afecto - el enamoramiento - el que hace surgir en él el deseo de alcanzar simultáneamente una identidad norteamericana para poder echar raíces en EEUU y dejarse 
absorber por su cultura. La prueba es que deja constancia de su vaivén en un libro escrito en inglés: Leaving.

Sin embargo, a veces le resulta difícil escribir en inglés lo que experimentó en español y, por eso, en medio del libro, en el capítulo central "Nombres y lenguas" (Leaving 87-91), inserta cinco páginas en castellano. Aunque estas cinco páginas son inaccesibles para el público estadounidense medio, son sintomáticas porque visibilizan el problema del lenguaje en el propio texto, como una escena de intraducibilidad radical.

Esto también lo demuestra el hecho de que, años más tarde, en 2010, el propio autor tradujo Leaving al español como Irse. En el prólogo a esta autotraducción, Waisman se empeña en evitar que esta versión española, elaborada a partir del inglés, sea percibida como una simple retraducción o back translation, en cuyo caso funcionaría como el verdadero original: hacer su novela accesible a un público argentino no debe equivaler a aniquilar la dimensión plurilingüe de la obra. Por eso insiste en el carácter paradójico de su autotraducción: lo que puede parecer una vuelta al origen ha resultado serlo solo en parte.

No es de extrañar que tanto Leaving como Irse hayan salido en editoriales independientes de California y Buenos Aires, respectivamente. Más que a un multilingüismo lexical, la complejidad de ambos textos se debe a la densidad de la reflexión en torno a los lazos entre lengua e identidad y al rechazo de un imaginario monolingüe, por lo que se aparta del esencialismo tan presente en los language memoirs norteamericanas. Para Waisman, la autotraducción constituye un acto vital que da forma a un espacio de mediación en el que el intercambio transcultural es posible y permite la fusión de sus identidades, lenguas y culturas. Al romper el supuesto vínculo orgánico entre experiencia y lengua, obras como la de Waisman, a su manera, contribuyen a instalar la condición postmonolingüe, ya que muestran una gran permeabilidad a la traducción. 


\section{Conclusiones: formas emergentes del postmonolingüismo}

La paradoja principal que se desprende de los casos estudiados es que no siempre se detecta en los textos bajo análisis el multilingüismo que se profesa en la crítica actual sobre la literatura latina en EEUU porque es juzgado éticamente deseable y políticamente correcto. Hay, por cierto, autores que instalan su obra en una "interlengua": son sobre todo escritores de origen caribeño como Díaz o Dicent, o resueltamente transnacionales como Chávez-Silverman. Pero en la mayoría de los casos, como se ha ilustrado con la autobiografía de Stavans, se trata de la variante "transparente", que no choca con las premisas unilingües de la industria editorial estadounidense. Hay más: la aparición de vocablos extranjeros y realia puede disfrazar una perspectiva estructuralmente unilingüe.

Por otra parte, si se toma en cuenta la trayectoria de un autor, pueden detectarse modalidades alternativas de multilingüismo, como el monolingüismo serial de autores que se autotraducen (Waisman). Y al nivel de la obra, mostrar la pluralidad de cada lengua, también del inglés, mediante operaciones de deconstrucción estilísticas, tal como lo hace Waisman en el seno de Leaving, constituye una subversión más profunda del monolingüismo. Siguiendo los aportes de Yildiz, cabe afirmar que las emergentes prácticas postmonolingües en la comunidad latina nos desafían a identificar otros mecanismos o inflexiones que expresen dislocamiento lingüístico y a trascender la oposición estándar entre el español y el inglés.

Otra paradoja tiene que ver con el género de la autobiografía. Aunque casi todos los textos del corpus incorporan reflexiones sobre el bilingüismo de los autores y su impacto en el proceso de escritura, dando muestras de lo que Lise Gauvin ha llamado una hiperconciencia lingüística (6-15), son menos multilingües de facto que muchas obras de ficción. Mientras que los novelistas a menudo tienden a evocar miméticamente la realidad representada, recurriendo abundantemente a ideolectos o sociolectos para caracterizar a sus personajes, los autores de autobiografías interculturales, viéndose 
afectados por la tensión entre resistencia y asimilación, no suelen elegir la vía de Waisman, sino la de Stavans. Antes bien, sus obras tratan de cerrar en cierta medida la brecha entre su yo dejado atrás y su nuevo yo. Aunque la tensión nunca puede eliminarse del todo y siempre queda algo de Unheimlich en ese espacio intersticial, el sujeto autobiográfico pone énfasis en la transformación que ha sufrido escribiendo en la lengua "postmaterna". La doble perspectiva y la autotraducción, que son parte esencial del proyecto artístico de cada autor multilingüe, suelen preceder a la escritura, de modo que las huellas lingüísticas de esa lucha a menudo dolorosa son menos visibles en los textos finales.

\section{Referencias}

Aparicio, Frances R. "U.S. Latino Expressive Cultures”. The Columbia History of Latinos in The United States since 1960. Edited by Gutiérrez, D. G. New York: Columbia University Press, 2004, pp. 355-390.

Apter, Emily. Against World Literature: On the Politics of Untranslatability. New York: Verso, 2013.

Braschi, Giannina. Yo-yo Boing!. Pittsburgh: Latin American Literary Review Press, 1998.

Bustamente, Fernanda E. A ritmo desenfadado: Narrativas dominicanas del nuevo milenio. Santiago de Chile/Santo Domingo: Cuarto Propio/Cielo Naranja, 2014.

Bruce-Novoa, Juan. Chicano Authors: Inquiry by Interview. Austin: University of Texas Press, 1980. 
Chávez-Silverman, Susana. Killer Crónicas: Bilingual Memories. Madison: University of Wisconsin Press, 2004.

Ch’ien, Evelyn. Weird English. Cambridge: Harvard University Press, 2004.

De Maeseneer, Rita. “ ‘Aprende el difícil’: Junot Díaz, Josefina Báez y las lecturas nacionales”. Pasavento. Revista de Estudios Hispánicos. II. 2, (2014): 345-357. Disponibilidad en: < http://www.pasavento.com/pdf/04_06_demaeseneer.pdf> . Acceso en: 12/12/2019.

Díaz, Junot. Drown. New York: Riverhead Books, 1996.

Díaz, Junot. The Brief Wondrous Life of Oscar Wao. New York: Riverhead Books, 2007.

Dicent, Juan. Winterness. Santo Domingo: Ediciones De a Poco, 2012.

Gauvin, Lise. "D'une langue à l'autre. La surconscience linguistique de l'écrivain francophone”. L'écrivain francophone à la croisée des langues. Entretiens, edited by Lise Gauvin, Paris: Karthala, 1997, pp. 6-15.

Grosjean, François. Life with Two Languages: An Introduction to Bilingualism. London: Harvard UP, 1982.

Gramling, David. The Invention of Monolingualism. New York: Bloomsbury, 2016.

Grutman, Rainier. "Multilingualism". Routledge Encyclopedia of Translation Studies, edited by Mona Baker and Gabriela Sadana. London: Routledge, 2009. p. 182-196.

Lennon, Brian. In Babel's Shadow: Multilingual Literatures, Monolingual States. Minneapolis: University Press of Minnesota, 2010.

Lipski, John M. Variación del español. Serie Cultura Hispánica, $\mathrm{n}^{\mathrm{o}}$ 10. Tokyo: Centro de Estudios Hispánicos Universidad Sofía, 2004. 
Loustau, Laura. Cuerpos errantes: literatura latina y latinoamericana en Estados

Unidos. Rosario: Beatriz Viterbo, 2002.

Paz Soldán, Edmundo; Fuguet, Alberto (Eds). Se habla español: voces latinas en USA. Madrid: Alfaguara, 2000.

Rodríguez, Juana María. "Latino, Latina, Latin@”. Keywords for American Cultural Studies, edited by Bruce Burgett and Glenn Hendler, New York: NYU Press, 2014. Disponibilidad en: < https://keywords.nyupress.org/americancultural-studies/author/jmrodriguez/ $>$. Accesso en: 12/12/2019.

Sandoval, Alberto; Aparicio, Frances R. "Hibridismos culturales: Literatura y cultura de los latinos en Estados Unidos". Revista Iberoamericana. LXXI.212, (2005): 665-697.

Sommer, Doris. Bilingual Aesthetics: A New Sentimental Education. Durham, NC: Duke University Press, 2004.

Stavans, Ilan. On Borrowed Words. A Memoir of Language. New York: Viking Penguin, 2001.

Tidigs, Julia; Huss, Markus. "The Noise of Multilingualism: Reader Diversity, Linguistic Borders and Literary Multimodality". Critical Multilingualism Studies. 5:1, (2017): 208-235.

Torres, Lourdes. "In the Contact Zone: Code Switching Strategies by Latino Writers”. Melus. 32.1, (2007):75-96.

Waisman, Sergio (a). Leaving. Oakland: Intelibooks, 2004.

Waisman, Sergio (b). Irse. Buenos Aires: Bajo la luna, 2010.

Waisman, Sergio (c). "Argentine Writers in the US: Writing South, Living North". A Companion to US Latino Literatures, edited by Carola Caulfield and Darién J. Davis, Woodbridge: Tamesis, 2007, pp. 158-176. 
Walkowitz, Rebecca. L. Born Translated: The Contemporary Novel in an Age of World Literature. New York: Columbia University Press, 2015.

Yildiz, Yasemin. Beyond the Mother Tongue: The Postmonolingual Condition. New York: Fordham University Press, 2012.

Recebido em: 28/02/2020 Aceito em: 27/04/2020 Publicado em julho de 2020

Ilse Logie. E-mail: ilse.logie@ugent.be. ORCID: https://orcid.org/0000-00027344-1793

Cad. Trad., Florianópolis, v. 40, $\mathbf{n}^{0}$ esp., p. 50-70, jan./jul. sem. 1. 2020.70 